C 1996 IEEE. Personal use of this material is permitted. However, permission to reprint/republish this material

for advertising or promotional purposes or for creating new collective works for resale or redistribution to servers

or lists, or to reuse any copyrighted component of this work in other works must be obtained from the IEEE.

\title{
RECENT PROGRESS ON BEAM STABILITY STUDY IN THE PSR ${ }^{*}$
}

\author{
T. Wang, P. Channell, R. Cooper, D. Fitzgerald, T. Hardek, R. Hutson \\ A. Jason, R. Macek, M. Plum, C. Wilkinson, and E. Colton ${ }^{\dagger}$ \\ Los Alamos National Laboratory, Los Alamos, New Mexico 87545
}

\begin{abstract}
A fast transverse instability has been observed in the Los Alamos Proton Storage Ring (PSR) when the injected beam intensity reaches more than $2 \times 10^{13}$ protons per pulse. Understanding the cause and control of this instability has taken on new importance as the neutron-scattering community considers the next generation of accelerator-driven spallation-neutron sources, which call for peak proton intensities of $10^{14}$ per pulse or higher. Previous observations and theoretical studies indicate that the instability in the PSR is most likely driven by electrons trapped within the proton beam. Recent studies using an experimental electronclearing system and voltage-biased pinger-electrodes for electron clearing and collection support this hypothesis. Experiments have also been performed to study the instability threshold when varying the electron production rate. Theoretical studies include a computer simulation of a simpli®ed model for the $e-p$ instability and the investigation of possible electron con${ }^{\circledR}$ nement in the ring-element magnetic ${ }^{\circledR}$ elds. This paper reports some recent results from these studies.
\end{abstract}

\section{INTRODUCTION}

The PSR is a fast-cycling high-current storage ring designed to accumulate beam over a macropulse of the LAMPF linac $(\sim 1$ $\mathrm{ms}$ ) by multi-turn injection through a stripper foil and compress that beam into a short single-turn extracted pulse $(\sim 0.25 \mu \mathrm{s})$, which drives a neutron source. Key PSR parameters include kinetic energy of $797 \mathrm{MeV}$, circumference of $90.1 \mathrm{~m}$, revolution frequency of $2.875 \mathrm{MHz}$, betatron tunes $\nu_{x}$ and $\nu_{y} \approx 3.17$ and 2.13 , respectively, and present operation with $N \approx 2.35 \times 10^{13}$ stored particles. The design intensity is $100 \mu \mathrm{A}$ on target at 12 $\mathrm{Hz}$, which implies $5.2 \times 10^{13}$ protons/pulse. Average and peak intensities have been somewhat less $\left(80 \mu \mathrm{A}\right.$ at $20 \mathrm{~Hz}$ and $4 \times 10^{13}$ maximum pulse size). The average current is limited by slow beam losses, and individual pulse intensities are limited by a fast instability [1-3].

The instability occurs when more than $\sim 2 \times 10^{13}$ protons are stored in bunched mode ( $\mathrm{rf}$ on), and when more than $\sim 5 \times 10^{12}$ are stored in unbunched mode. Transverse oscillations at $\sim 100$ $\mathrm{MHz}$ are seen, and grow exponentially at time scales of $10 \pm 100$ $\mu \mathrm{s}$, causing beam losses. Searches for a possible impedance source were unsuccessful. Much evidence has been observed which supports the theory that the instability is caused by the coupled oscillation between the proton beam and trapped electrons \pm the ${ }^{\mathrm{a}} e-p^{\mathrm{o}}$ instability that has been previously observed in some other proton facilities [4-6]. Supporting observations include the following: degrading the vacuum makes the beam

\footnotetext{
*supported by Los Alamos National Laboratory Institutional Supporting Research under the auspices of the United States Department of Energy.

tUS DOE.
}

become more unstable, biasing the foil to a voltage suf®cient to clear electrons in the vicinity increases the stability threshold; and moving halo scrapers into the beam pipe to produce more secondary electrons decreases the threshold. Theoretical calculations have also shown that the conditions for an $e-p$ instability may occur in the PSR. However, a dominant electron source has not yet been identi ${ }^{\circledR e d}$. Understanding of this instability and methods of controlling it have taken on new importance as the neutron scattering community considers the next generation of accelerator-driven spallation-neutron sources, which call for peak proton intensities of $\sim 2 \times 10^{14}$ per pulse or higher.

A theory proposed in the early nineties conjectured that a small amount of the beam in the PSR may leak into the gap to form a smooth, overall density distribution and an electric potential suf®cient for electron trapping to cause instability $[1,7]$. A later computer simulation indicated that neither the multi-turn trapping of electrons nor the gap-®lling is necessary for the $e$ $p$ instability to develop if there are suf®cient electrons trapped in the proton bunch, but the instability threshold can be lowered by having electrons trapped for more than one turn $[3,8]$. Observations did show that the instability is associated with bunch leakage; with bunched beam ( $\mathrm{rf}$ on), we observed that instability occurs when the inter-bunch gap has $® l l e d$ in. Measurements taken under various conditions indicate that gap ®1ling occurs either before or simultaneously with the beginning of growing oscillations.

In the recent years, experiments were performed by using the ${ }^{a}$ pinger $^{\circ}$ to sweep the leakage out of the inter-bunch gap during storage and by lowering the machine transition gamma to prevent protons to make leakage $\operatorname{dif} ®$ cult [3]. In the relatively lowintensity regime, results from these experiments seem to con ${ }^{\circledR} \mathrm{rm}$ the conjecture that the gap leakage does induce the instability in the PSR. Yet at high beam intensity, neither the gap sweeping nor the low transition gamma was able to stabilize the beam. One possible explanation is that keeping the gap absolutely clean at high beam intensity is dif®cult. However, the results of these experiments can not exclude other mechanisms to drive the instability; whether the beam in PSR will be stable with a clean gap at the intensity of $5.2 \times 10^{13}$ protons/pulse remains to be resolved, and identifying the source of electrons still needs further study.

Recently experimental studies of beam stability in the PSR were carried out by using a newly installed electron clearing system and by bumping the beam close to the wall to create electrons that may induce the instability. Our recent theoretical study covers a simple simulation of the $e-p$ instability, a study of possible electron trapping in the magnetic ®eld of the focusing elements, and an estimation of the secondary emission of electrons from protons lost in the ring. This paper reports some of the progress made in these recent studies. 


\section{EXPERIMENTAL OBSERVATIONS}

\section{A. Electron Clearing}

Since a charge-change injection method with foil stripping is used in the PSR, we believed that the injection section should have more electrons than other part of the ring. Recently an experimental clearing system, including two pairs of electrodes, two bending magnets for directing the convoy electrons from the stripping process, and an injection foil biasing capability, was implemented in this section to study the effect of electron clearing on the beam stability. The system setup and the experimental details are reported in a separate paper [9].

The results of applying this clearing system to the beam apparently depends on the machine and beam conditions. In the most effective case, we have observed a more than $20 \%$ increase of the instability threshold current of a continuous coasting beam. In this case, we only applied moderate biasing voltages on the foil and clearing electrodes. For bunched beams, we have tried higher voltages on the foil and the clearing electrodes; we found no signi ${ }^{\circledR}$ cant effect on the instability threshold, except that the growth rate of the instability was lowered. A lack of suf®cient machine development time did not allow more thorough studies on the bunched beam. Nonetheless, the results of this experiment do provide strong support to the $e-p$ assumption.

\section{B. Electron Collection}

We performed the experiments of electron collection by using the plates of the clearing electrodes in the injection Section and the pinger in Section 3 as electron collectors. Synchronized signals from the collectors and the wall current monitor or the BPM were recorded and studied.

In the coasting-beam experiments, we observed a large current collected by the pinger electrodes during the later stage of the storage. When the stored beam went unstable, we observed that the loss of beam current was preceded by a sudden rise in the pinger current. In the bunched mode, when the stored beam was marginally stable, we found the collected currents from the pinger and from the clearing electrodes increased with the amplitude of the vertical oscillation. For unstable bunched beams, we observed that the loss of beam current is always accompanied by a fast increase in the current collected on the pinger electrodes.

\section{Beam Bump Experiment}

The purpose of this experiment was to prove that an excessive amount of the secondary electrons generated by the protons lost to the wall can induce the $e-p$ instability. A similar experiment was carried out some years ago by moving a beam scraper close to the core of the beam to generate secondary electrons to induce the instability.

We created a horizontal beam bump in Section 3 of the ring where a pinger was used as an ion chamber to collect the secondary electrons. A fast instability was observed when the orbit was bumped to more than a half centimeter toward the ring center. When we bumped the orbit away from the ring center, the stored beam remained stable up to more than one centimeter of bump. The pinger collected a substantial amount of current when the beam was stable, and a large amount of current when the beam was unstable. The stability property of the beam observed in this experiment is consistent with results of the beam scraping experiment before.

\section{THEORETICAL STUDY}

\section{A. Instability Simulation}

A simple computer simulation of the e-p instability in a long proton bunch has been performed by numerically solving the equations of motion for the centroid of the proton beam and the centroid of the trapped electrons. A preliminary result has been reported earlier. Recent studies include using re®ned linedensity models, investigating the effects of the AG focusing and noise, and a study of the BPM signal. Details are documented in a separate paper [8] and in an internal report [10].

\section{B. Electron Trapping Study [11]}

The partial success in stabilizing the beam by gap sweeping with the pinger motivated the search for other mechanisms besides gap-®lling, for multi-turn trapping of electrons in the PSR. A recent study investigated the possibility that electrons could be trapped in the ®eld of lattice quadrupoles. Computer tracking of the electron motion in the combined quadrupole and beam ${ }^{\circledR}$ elds indicates that a fraction of the electrons generated in the quadrupole region, including the gas ionization and secondary emission, can be trapped in the quadrupole ®eld over several turns. The total amount of electrons trapped in all the quadrupoles can possibly reach a few percent of the amount of the protons stored, enough to trigger the $e-p$ instability in the PSR.

\section{Study on Possible Electron Source [12]}

We have studied the possibility of secondary electrons generated by the protons lost to walls as an electron source in the PSR. In this estimate, we applied Sternglass' theory [13] on the yield of secondary emission. We found that the yield for the secondary emission in the PSR, due to a proton lost to the beam pipe, is about 200. Using the experimental data of beam loss in the PSR, and the theoretically estimated yield, we inferred that the electrons generated in one accumulation cycle in a typical PSR production condition is about $230 \mathrm{nC}$. The charge collected by using the pinger as an ion chamber, ranges from 90 to $550 \mathrm{nC}$. Thus, the estimated electron production is in the range of the measured data. This implies that the secondary emission due to the lost proton can be a signi ${ }^{\circledR}$ cant electron source in the PSR.

\section{SUMMARY AND CONCLUSIONS}

Our recent experiments included electron clearing in the injection section, electron collection, and beam bumping for inducing the instability. Our recent theoretical study covers instability simulation, a study of multi-turn electron trapping in the quadrupole ${ }^{\circledR}$ led, and a study of secondary emission as a possible electron source. Results from our recent experimental and theoretical studies further con®rm that the observed instability in the PSR is an $e-p$ instability. Based on these observations and the results from other experiments as well as theoretical estimations, we tend to conclude that secondary emission could be one of the major electron sources in the PSR. If this is true, a hypothesis for 
creating the large amount of electrons collected in the PSR can be conjectured: Initially, any small perturbation can start a relative oscillation between the injected protons and the electrons created due to injection, gas ionization, and secondary electrons produced by lost protons. This oscillation together with the increasing beam emittance due to the repetitive scattering by the injection foil cause proton loss and the production of more secondary electrons around the ring. These secondary electrons, in turn can increase the beam oscillation to cause more proton loss. Thus, the beam oscillation and the secondary emission of electrons due to the lost protons mutually enhance each other to increase the amount of electrons in the ring above the threshold of the $e-p$ instability.

We plan to upgrade the beam injection section of the PSR to improve the quality of the injected proton beam for reducing the proton loss and machine activation. This upgrade may change the characteristics of the beam stability in the PSR and lead to a better understanding of the instability. Understanding this instability and methods of controlling it have fundamental importance in both the future operation of the PSR and the design of the next generation of accelerator-driven spallation-neutron sources. Study of the PSR instability will be continued in the future.

\section{References}

[1] D. Neuffer, Conf. Record of the 1991 IEEE Particle Accelerator Conf., Vol. 2, p. 1077, and references cited therein.

[2] D. Neuffer et al., Nucl. Instr. and Meth., A321, 1 (1992).

[3] T. Wang et al., Conf. Record of the 1993 IEEE Particle Accelerator Conf., Vol. 3, p. 1896.

[4] H. Grunder and G. Lambertson, Proc. 8th Intl. Conf. on High Energy Accelerators, CERN, 1971, p. 308.

[5] H. G. Hereward, CERN Internal Report 71-15 (1971).

[6] H. Schìnnauer and B. Zotter, CERN Internal Note, May 1972.

[7] D. Neuffer and C. Ohmori., Nucl. Instr. and Meth., $\underline{\mathbf{A 3 4 3}}$, 390 (1994).

[8] T. Wang et al., these proceedings.

[9] M. Plum et al., these proceedings.

[10] T. F. Wang, Los Alamos Accelerator Theory Note, in preparation.

[11] P. Channell and A. Jason, AOT-3 Technical Note: 93-11, AOT Division, Los Alamos National Laboratory, 1993.

[12] M. Plum, Los Alamos PSR Technical Note, PSR-95001,1995.

[13] E. J. Sternglass, Phys. Rev. 108, 1 (1957). 